\title{
A Case with XXXX Syndrome Who Was Incidentally Diagnosed during Examination for Suspected Post-Human Papillomavirus Vaccination Syndrome
}

\author{
Akiyo Hineno1,2, Tomoki Kosho ${ }^{3}$, Hiroyuki Kato ${ }^{4}$, Yoshiki Sekijima ${ }^{2}$, Shu-ichi Ikeda ${ }^{{ }^{*}}$ \\ ${ }^{1}$ Intractable Disease Care Center, Shinshu University Hospital, Matsumoto, Japan \\ ${ }^{2}$ Department of Medicine (Neurology and Rheumatology), Shinshu University School of Medicine, Matsumoto, Japan \\ ${ }^{3}$ Department of Medical Genetics, Shinshu University Hospital, Matsumoto, Japan \\ ${ }^{4}$ Department of Orthopaedic Surgery, Shinshu University School of Medicine, Matsumoto, Japan \\ Email: *ikedasi@shinshu-u.ac.jp
}

How to cite this paper: Hineno, A., Kosho, T., Kato, H., Sekijima, Y. and Ikeda, S. (2019) A Case with XXXX Syndrome Who Was Incidentally Diagnosed during Examination for Suspected Post-Human Papillomavirus Vaccination Syndrome. Case Reports in Clinical Medicine, 8, 239-244. https://doi.org/10.4236/crcm.2019.89029

Received: August 15, 2019

Accepted: September 3, 2019

Published: September 6, 2019

Copyright $\odot 2019$ by author(s) and Scientific Research Publishing Inc. This work is licensed under the Creative Commons Attribution International License (CC BY 4.0).

http://creativecommons.org/licenses/by/4.0/

\begin{abstract}
A 19-year-old Japanese woman was referred to us with the complaints of arthralgia and meralgia following human papillomavirus (HPV) vaccination. She received HPV vaccination at the age of 15 years and three years later, she developed intermittent arthralgia, meralgia, and numbness in limbs. There were no orthostatic dysregulation symptoms. She had hypertelorism, and brachydactyly in both the hands, and revealed mild cubitus varus deformity with lateral instability. X-ray examination disclosed hypoplasia of the humeral capitellum and trochlea in elbow joints. G-banded chromosomes were shown to be composed of 48, XXXX. She was, therefore, diagnosed with XXXX syndrome, which explained the reason for her limb symptoms. Although some girls with HPV vaccination complain of various symptoms including limb pain and numbness, exact underlying cause of these symptoms needs to be ascertained carefully for reaching a final diagnosis.
\end{abstract}

\section{Keywords}

XXXX Syndrome, Tetrasomy X, Human Papillomavirus Vaccine, Arthralgia, Complex Regional Pain Syndrome

\section{Introduction}

Human papillomavirus (HPV) vaccine was introduced for the prevention of uterine and cervical cancer [1]. Subsequently, in several countries, a significant num- 
ber of adolescent girls with HPV vaccination complained of possible adverse effects, such as general fatigue, chronic headache, arthralgia, limb pain and weakness, and difficulty in learning [2]-[9]. This post-HPV vaccination syndrome is explainable by a combination of orthostatic dysregulation, chronic regional pain syndrome (CRPS), and brain cognitive dysfunction [10]. Additionally, some patients reported having developed other diseases after HPV vaccination [11] [12]; however, the relationship between HPV vaccination and the onset of other diseases remains unclear.

Here, we report a case wherein the patient was diagnosed with XXXX syndrome during examination for suspected post-HPV vaccination syndrome. We obtained the patient's consent for publishing this case report.

\section{Case Report}

A 19-year-old Japanese woman was referred to our hospital with a compliant of persistent joint pain, especially with both the elbows. She had a history of postural hand tremor and her father experienced a similar disorder. Further, she reported of a delay in verbal development, learning disability, school refusal, and had been suffering from depression requiring multidrug therapy during childhood. She received three injections of HPV vaccine at the age of 15 years; the first dose of HPV vaccine (Cervarix $\left.{ }^{\circledR}\right)$ in September 2011 and third dose in March 2012. After three years, she developed intermittent meralgia, arthralgia, and numbness in the limbs, which occasionally hindered her daily activities. Although she visited several hospitals with her mother, her diagnosis was obscure. Finally, her mother suspected that these symptoms were related to HPV vaccination and thus, they consulted us in November 2015.

On examination, she was $172.0 \mathrm{~cm}$ tall and weighed $51 \mathrm{~kg}$. She had hypertelorism, midfacial hypoplasia, a high palate, and bilateral brachydactyly with nail hypoplasia (Figure 1(A)). Both the elbows had lateral instability with cubitus varus deformity in the elbow joints bilaterally, which was revealed upon elevating the arms (Figure 1(B)). However, neither swelling nor redness was seen in any of these joints. X-ray examination indicated hypoplasia of the humeral capitellum and trochlea in both the elbow joints (Figure 2). Routine laboratory tests did not reveal any abnormal findings. The serum C-reactive protein and anti-matrix metalloproteinase (MMP) levels were $0.04 \mathrm{mg} / \mathrm{dl}$ and $13.2 \mathrm{ng} / \mathrm{ml}$ (normal: 17.3 - $59.7 \mathrm{ng} / \mathrm{ml}$ ), respectively. The results of antibody tests for rheumatoid factor and anti-cyclic citrullinated peptide (CCP) antibodies were also
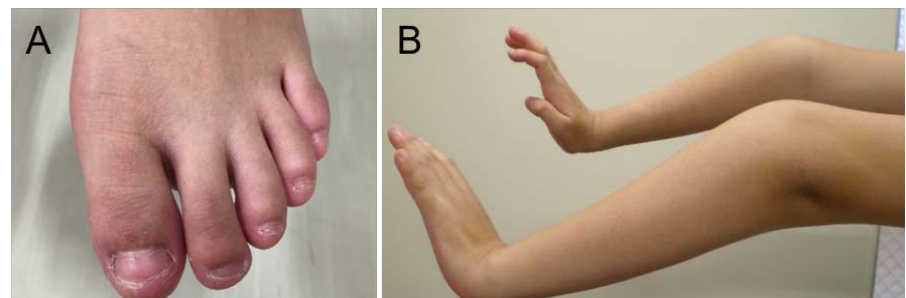

Figure 1. Brachydactyly in foot (A) and cubitus varus (B). 
negative. G-banded chromosomal analysis disclosed 48, XXXX (Figure 3), and she was diagnosed with XXXX syndrome. The patient underwent regular medical examinations in neurology, orthopedic surgery, and genetic counseling departments. Elbow supporters, analgesic drugs, and rehabilitation effectively reduced patient's limb pain and psychosocial support relieved her mother's anxiety. The patient continues to receive rehabilitation and did not require any pain-relieving medication in 2018.
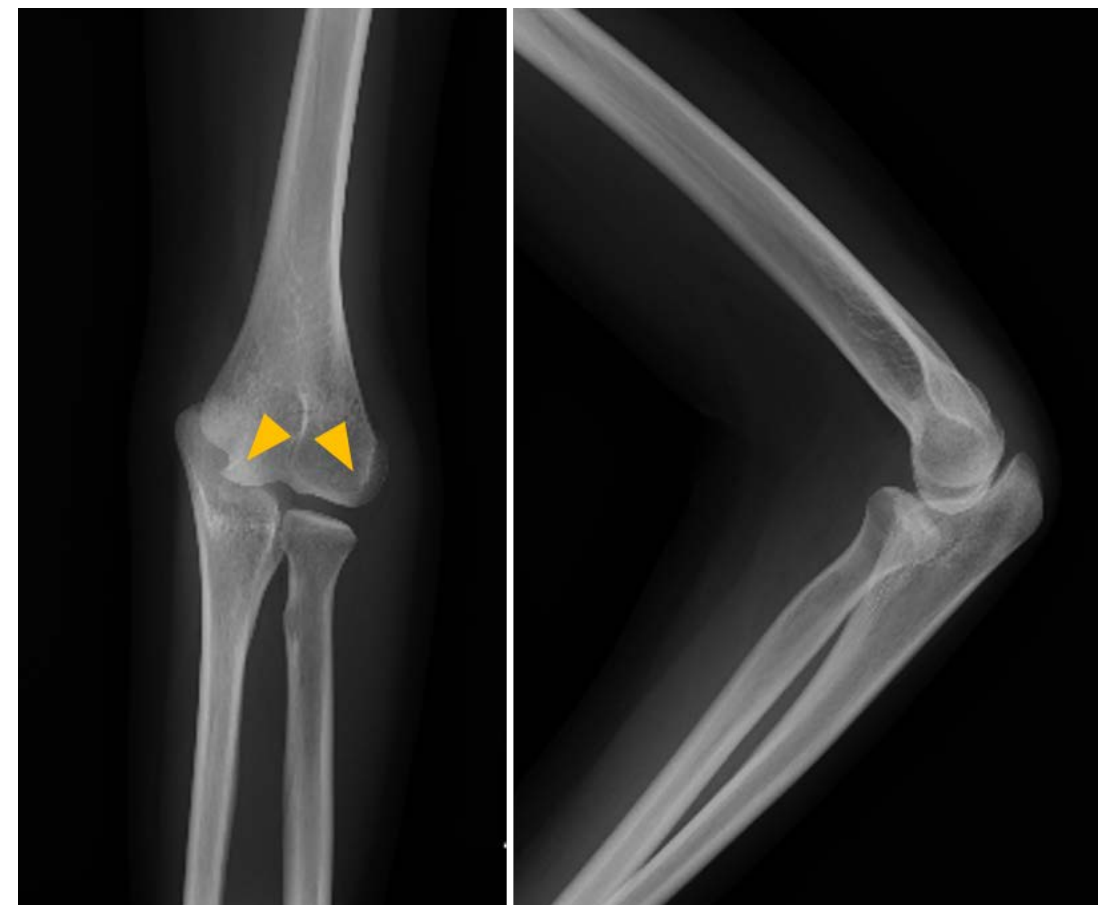

Figure 2. X-ray of the elbow showing hypoplasia of the humeral capitellum and trochlea (arrowheads).
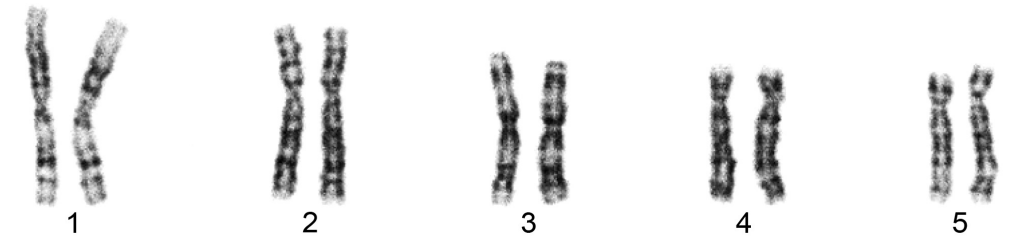

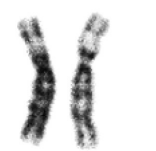

6

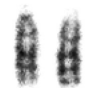

13

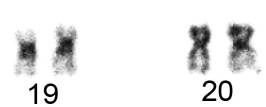

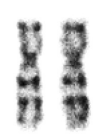

7

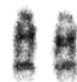

14

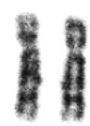

8

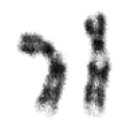

9

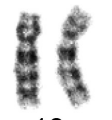

10

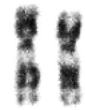

11

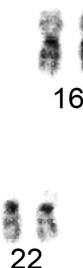

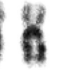

16

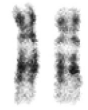

12

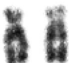

18

Figure 3. G-banded chromosomal analysis showing 48, XXXX. 


\section{Discussion}

XXXX syndrome is a rare sex chromosomal abnormality, which is characterized by tall stature, facial dysplasia (midfacial hypoplasia, hypertelorism) and limb deformity (radioulnar synostosis), intellectual disability, and speech and behavioral problems [13]. Cases with polysomy $\mathrm{X}$ accompanied with systematic lupus erythematosus (SLE) were reported and it is likely that X-chromosome polysomy plays a role in higher incidence of autoimmune diseases [14]. Contrarily, it is also surmised that HPV vaccination can induce autoimmune diseases, including SLE and rheumatoid arthritis [8].

Our present case developed arthralgia and meralgia of unknown cause, three years after HPV vaccination. We previously reported patients with arthritis after HPV vaccination [15]. Incubation period of three years is rather long to consider a post-HPV vaccination syndrome as a cause for her limb pain [3], and clinical manifestations without any orthostatic dysregulation symptoms were atypical for this syndrome [2] [10]. The patient was accidentally diagnosed with XXXX syndrome and we hypothesized that this congenital disorder may be responsible for her limb pain including arthralgia and meralgia. Although some cases with polysomy X have been reported, most of them without SLE did not complain of pain including arthralgia [14] [16] [17]. Recent evidence has shown that autoantibodies against adrenergic and cholinergic receptors play an important role in the appearance of both orthostatic dysregulation and CRPS [18] [19]. The relationship between HPV vaccination and positive serum autoantibodies against autonomic nerve receptors have already been described in the previous three cases [20] [21] [22]. Thus, it cannot be completely ruled out that HPV vaccination worked as a trigger for inducing limb pain. Finally, we emphasize that it is very important to carefully check for other disorders, such as a congenital disorder, during examination of girls complaining of various symptoms after HPV vaccination.

\section{Conclusion}

A case with a rare congenital disorder, XXXX syndrome, was reported. Although the patient was initially suspected to be suffering from post-HPV vaccination syndrome, careful physical examination and chromosomal analysis led to the final diagnosis of XXXX syndrome. Suspected adverse effects following HPV immunization are complex conditions which might be due to certain disorders. A thorough investigation is required during examination of the patients with suspected post-HPV vaccination syndrome.

\section{Funding}

This study was supported by a grant from a Health and Labour Science Research Grant on Emerging and Re-emerging Infectious Diseases (Establishment of Treatments and Providing Information on Symptoms after HPV vaccination, H28-001) to S.I. from the Ministry of Public Heath, Labour and Welfare, Japan. 


\section{Conflicts of Interest}

The authors declare no conflicts of interest regarding the publication of this paper.

\section{References}

[1] Future II Study Group (2007) Quadrivalent Vaccine against Human Papillomavirus to Prevent High-Grade Cervical Lesions. The New England Journal of Medicine, 356, 1915-1917. https://doi.org/10.1056/NEJMoa061741

[2] Kinoshita, T., Abe, R.T., Hineno, A., Tsunekawa, K., Nakane, S. and Ikeda, S.I. (2014) Peripheral Sympathetic Nerve Dysfunction in Adolescent Japanese Girls Following Immunization with the Human Papillomavirus Vaccine. Internal Medicine, 53, 2185-2200. https://doi.org/10.2169/internalmedicine.53.3133

[3] Ozawa, K., Hineno, A., Kinoshita, T., Ishihara, S. and Ikeda, S.I. (2017) Suspected Adverse Effects after Human Papillomavirus Vaccination: A Temporal Relationship between Vaccine Administration and the Appearance of Symptoms in Japan. Drug Safety, 40, 1219-1229. https://doi.org/10.1007/s40264-017-0574-6

[4] Brinth, L., Theibel, A.C., Pors, K. and Mehlsen, J. (2015) Suspected Side Effects to the Quadrivalent Human Papillomavirus Vaccine. Danish Medical Journal, 62, A5064.

[5] Blitshteyn, S. (2014) Postural Tachycardia Syndrome Following Human Papillomavirus Vaccination. European Journal of Neurology, 21, 135-139.

https://doi.org/10.1111/ene.12272

[6] Brinth, L.S., Pors, K., Theibel, A.C. and Mehlsen, J. (2015) Orthostatic Intolerance and Postural Tachycardia Syndrome as Suspected Adverse Effects of Vaccination against Human Papillomavirus. Vaccine, 33, 2602-2605. https://doi.org/10.1016/j.vaccine.2015.03.098

[7] Martínez-Lavín, M. (2014) Fibromyalgia-Like Illness in 2 Girls after Human Papillomavirus Vaccination. Journal of Clinical Rheumatology, 20, 392-393.

[8] Bharti, V.K. and Srivastava, R.S. (2009) Protective Role of Buffalo Pineal Proteins on Arsenic-Induced Oxidative Stress in Blood and Kidney of Rats. Health, 1, 167-172. https://doi.org/10.4236/health.2009.13027

http://www.scirp.org/fileOperation/downLoad.aspx?path=Health20090100017_971 88589.pdf\&type=journal

[9] Palmieri, B., Poddighe, D., Vadala, M., Laurino, C., Carnovale, C. and Clementi, E. (2017) Severe Somatoform and Dysautonomic Syndromes after HPV Vaccination: Case Series and Review of Literature. Immunologic Research, 65, 106-116. https://doi.org/10.1007/s12026-016-8820-Z

[10] Blitshteyn, S., Brinth, L., Hendrickson, J.E. and Martinez-Lavin, M. (2018) Autonomic Dysfunction and HPV Immunization: An Overview. Immunologic Research, 66, 744-754. https://doi.org/10.1007/s12026-018-9036-1

[11] Gatto, M., Agmon-Levin, N., Soriano, A., Manna, R., Maoz-Segal, R., Kivity, S., et al. (2013) Human Papillomavirus Vaccine and Systemic Lupus Erythematosus. Clinical Rheumatology, 32, 1301-1307. https://doi.org/10.1007/s10067-013-2266-7

[12] Geier, D.A. and Geier, M.R. (2017) Quadrivalent Human Papillomavirus Vaccine and Autoimmune Adverse Events: A Case-Control Assessment of the Vaccine Adverse Event Reporting System (VAERS) Database. Immunologic Research, 65, 46-54. https://doi.org/10.1007/s12026-016-8815-9

[13] Jones, K.L. (2013) XXX and XXXX Syndromes. In: Jones, K.L., Jones, M.C. and del 
Campo, M., Eds., Smith's Recognizable Patterns of Human Malformation, 7th Edition, Elsevier Saunders, Philadelphia, 74-75.

[14] Slae, M., Heshin-Bekenstein, M., Simckes, A., Heimer, G., Engelhard, D. and Eisenstein, E.M. (2014) Female Polysomy-X and Systemic Lupus Erythematosus. Seminars in Arthritis and Rheumatism, 43, 508-512. https://doi.org/10.1016/j.semarthrit.2013.07.014

[15] Abe, R., Kinoshita, T., Hineno, A. and Ikeda, S. (2016) Monoarthropathy or Polyarthritis in Adolescent Japanese Girls Who Received Immunization with the $\mathrm{Hu}$ man Papillomavirus Vaccine. Case Reports in Clinical Medicine, 5, 109-114. https://doi.org/10.4236/crcm.2016.53020

[16] Tartaglia, N.R., Howell, S., Sutherland, A., Wilson, R. and Wilson, L. (2010) A Review of Trisomy X (47,XXX). Orphanet Journal of Rare Diseases, 5, 8. https://doi.org/10.1186/1750-1172-5-8

[17] Kara, C., Üstyol, A., Yılmaz, A., Altundağ, E. and Oğur, G. (2014) Premature Ovarian Failure Due to Tetrasomy X in an Adolescent Girl. European Journal of Pediatrics, 173, 1627-1630. https://doi.org/10.1007/s00431-013-2209-y

[18] Yu, X., Stavrakis, S., Hill, M.A., Huang, S., Reim, S., Li, H., et al. (2012) Autoantibody Activation of Beta-Adrenergic and Muscarinic Receptors Contributes to an "Autoimmune" Orthostatic Hypotension. Journal of the American Society of Hypertension, 6, 40-47. https://doi.org/10.1016/j.jash.2011.10.003

[19] Kohr, D., Singh, P., Tschernatsch, M., Kaps, M., Pouokam, E., Diener, M., et al. (2011) Autoimmunity against the $\beta 2$ Adrenergic Receptor and Muscarinic-2 Receptor in Complex Regional Pain Syndrome. Pain, 152, 2690-2700. https://doi.org/10.1016/j.pain.2011.06.012

[20] Blitshteyn, S. and Brook, J. (2017) Postural Tachycardia Syndrome (POTS) with Anti-NMDA Receptor Antibodies after Human Papillomavirus Vaccination. Immunologic Research, 65, 282-284. https://doi.org/10.1007/s12026-016-8855-1

[21] Hendrickson, J.E. and Tormey, C.A. (2016) Human Papilloma Virus Vaccination and Dysautonomia: Consideration for Autoantibody Evaluation and HLA Typing. Vaccine, 34, 4468. https://doi.org/10.1016/j.vaccine.2016.05.029

[22] Schofield, J.R. and Hendrickson, J.E. (2018) Autoimmunity, Autonomic Neuropathy, and the HPV Vaccination: A Vulnerable Subpopulation. Clinical Pediatrics, 57, 603-606. https://doi.org/10.1177/0009922817728701 